\title{
Superspace formulation of exotic supergravities in six dimensions
}

\section{Martin Cederwall}

Department of Physics, Chalmers University of Technology,

Gothenburg SE 412 96, Sweden

E-mail: martin.cederwall@chalmers.se

Abstract: We provide a linearised superfield description of the exotic non-metric $N=$ $(4,0)$ supergravity in $D=6$, by using a pure spinor superfield formalism. The basic field $\Psi$ is a ghost number 2 scalar, transforming in the same R-symmetry module as the tensor fields. Partial results for the $N=(3,1)$ model are presented.

KEYWORDS: Extended Supersymmetry, Supergravity Models

ARXIV EPRINT: 2012.02719 


\section{Contents}

1 Introduction 1

$2 N=(4,0) \quad 1$

$3 \quad N=(3,1)$ and $N=(3,0) \quad 6$

4 Conclusions $\quad 8$

\section{Introduction}

The existence of certain exotic gravity-like, but non-metric, supermultiplets in six dimensions was established by Strathdee [1] and used by Hull [2, 3] to build the physical models. The models are chiral, with $N=(4,0)$ or $(3,1)$ supersymmetry, reduce to ordinary supergravity in $D=5$, and are suspected to be related to strong coupling limits of $D=5$ supergravity. These models have received a renewed interest during the last few years [48], concerning for example Lagrangian formulations, supersymmetry and the possibility to formulate the models in terms of $E_{6}$ extended geometry $[9,10]$. Interacting versions of these models are still unknown, and should not be local field theories.

The purpose of the present letter is to present a superfield formulation. The main focus is on the $N=(4,0)$ model, and partial results are given for the $N=(3,1)$ model. The method used is "pure spinor superfield theory" [11-22]. It should be stressed that this formalism, which in addition to the standard superspace coordinates $Z^{M}=\left(x^{m}, \theta^{\mu}\right)$ involve constrained bosonic spinor ghosts, is nothing exotic, or a choice that may be avoided. Any supermultiplet has a natural formulation in terms of a pure spinor superfield, and the cohomological equations at a given ghost number are the standard superspace equations. However, with the inclusion of a constrained spinor, there is the advantage of treating all ghost numbers at once, i.e., fields, gauge symmetries, equations of motion (for maximal supersymmetry) etc. They arise collectively from a single field. In addition, it often provides a unique way of writing an action, even for maximally supersymmetric models like $D=11$ supergravity $[16,17]$. In the present case, we are dealing with chiral theories, and the pure spinor superfield formalism will not yield an action.

\section{$2 \quad N=(4,0)$}

A superfield formulation of the $D=6, N=(4,0)$ theory should be based on a "pure spinor" $\lambda$ in (001)(1000) of $D_{3} \oplus C_{4}$, the same module as the fermionic coordinates $\theta$. (We are working in a flat Minkowski background, so the fermionic variables are spinors.) It should obey the generic type of constraint $\left(\lambda \gamma^{a} \lambda\right)=0$, which means that the remaining modules in 

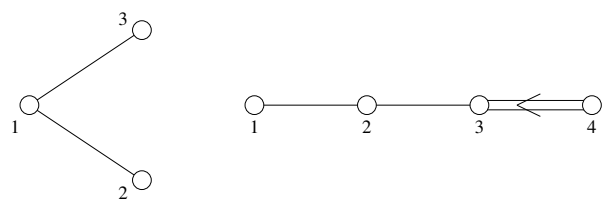

Figure 1. The Dynkin diagram, with our conventions for numbering of the nodes, of $D_{3} \oplus C_{4} \simeq$ $\mathfrak{s o}(6) \oplus \mathfrak{s p}(8)$.

$\lambda^{2}$ are $(002)(2000) \oplus(100)(0100) . \quad \lambda$ is not in a minimal orbit, but an intermediate one; this is normal, and happens e.g. in $D=11$ supergravity $[16,17]$. We still use the term "pure spinor", by need to keep in mind that it differs from a pure spinor in the sense of Weyl, which by definition lies in a minimal orbit. The conventions for the Dynkin labels (i.e., for the ordering of the nodes in the Dynkin diagram for $D_{3} \oplus C_{4}$ is the standard one, with $(100)(0000)$ being the Spin $(1,5)$ vector. The 32-dimensional chiral spinors in (010)(1000) and (001)(1000) are real through a USp(8)-Majorana condition, which is most easily seen using a quaternionic language, where $\mathfrak{s o}(1,5) \simeq \mathfrak{s l}(2 ; \mathbb{H})$ and $\mathfrak{u s p}(8) \simeq \mathfrak{a}_{4}(\mathbb{H})$, the algebra of $4 \times 4$ antihermitean quaternionic matrices. Then the spinors are $2 \times 4$ quaternionic matrices, acted on by $\mathfrak{s l}(2 ; \mathbb{H})$ from the left and $\mathfrak{a}_{4}(\mathbb{H})$ from the right.

We want to formulate the on-shell fields as cohomology of the BRST operator

$$
Q=\lambda^{\alpha} D_{\alpha}
$$

in some field $\Psi(x, \theta, \lambda)$. Here, $D_{\alpha}$ is the fermionic covariant derivative, whose torsion in flat superspace is given by the gamma matrices. The BRST operator is nilpotent due to the constraint on $\lambda$. However, the pure spinor constraint is irreducible, and removes 6 of the 32 degrees of freedom in $\lambda$. This implies that the cohomology of a scalar field becomes trivial. The zero-mode cohomology (i.e., the algebraic cohomology when $\frac{\partial}{\partial x}=0$ ) only consists of forms,

$$
\Psi_{0}(\theta, \lambda)=\sum_{n=0}^{6}\left(\lambda \gamma^{a_{1}} \theta\right) \ldots\left(\lambda \gamma^{a_{n}} \theta\right) A_{a_{1} \ldots a_{n}}
$$

The full cohomology becomes de Rahm cohomology. Even if the field $\Psi$ has no local degrees of freedom, it may play a rôle in an interacting theory, like e.g. is refs. [14, 15, 19].

The partition function of the pure spinor can be given explicitly. A partition only counting the number of functions at each power of $\lambda$ is

$$
\begin{aligned}
Z_{\lambda}(t)= & 1+32 t+522 t^{2}+5792 t^{3}+49207 t^{4}+341568 t^{5}+2018524 t^{6}+10447424 t^{7} \\
& +48349899 t^{8}+203253024 t^{9}+785789394 t^{10}+\ldots \\
= & \frac{(1+t)^{6}}{(1-t)^{26}}
\end{aligned}
$$

reflecting the number of degrees of freedom (26) in the pure spinor. A refined partition function, where the coefficients in the power expansion take values in the representation 
ring, is

$$
\begin{aligned}
\mathscr{Z}_{\lambda}(t) & =\frac{\bigoplus_{i=0}^{6} \wedge^{i}(100)(0000) t^{2 i}}{(1-t)^{(001)(1000)}} \\
& =(1-t)^{-(001)(1000)}\left(1-t^{2}\right)^{(100)(0000)} .
\end{aligned}
$$

Here, we have used the shorthand " $\left(1-t^{k}\right)^{ \pm R}$ " for the partition function of a bosonic (minus sign) or fermionic (plus sign) variable at level $k$. There are (at least) two ways to read this expression. One is to understand the numerator in the first expression as the zero-mode cohomology, the differential forms of eq. (2.2). The other reads the last expression as the product of the partition function for an unconstrained spinor and the partition function of the fermionic ghost for the constraint in (100)(0000). Since the constraint is irreducible, there are no further ghosts.

The latter reading displays the Koszul duality [20] of the pure spinor to a superalgebra $\mathscr{A}$ through the relation [23]

$$
\mathscr{Z}_{\lambda}(t) \otimes \mathscr{Z}_{\mathscr{A}^{+}}(t)=1 .
$$

Here, $\mathscr{Z}_{\mathscr{A}^{+}}$is the partition function for the (universal enveloping algebra of) the positive level subalgebra $\mathscr{A}^{+} \subset \mathscr{A}$. The superalgebra $\mathscr{A}$ is a Borcherds superalgebra, but since it is finite-dimensional (due to finite reducibility), it is also a finite-dimensional contragredient Lie superalgebra. In the present case, we obtain $\mathscr{A} \simeq \mathfrak{o} \mathfrak{s p}(8 \mid 8)$, the superconformal algebra of an $N=(4,0)$ theory in $D=6$. Similar appearances of the superconformal algebra seem to be typical for superconformal systems in a pure spinor superfield approach [14, 15, 19, 24].

One needs a field in some non-trivial module. In addition, it should be subject to some relation involving $\lambda$, so that the cohomology not just becomes the tensor product of the module of the field with the (trivial) cohomology of a scalar field. Here, it is helpful to remember that all fields, including ghosts and antifields, will appear as zeromode cohomology. The module of the field itself will coincide with that of the ghosts with highest ghost number. In the $(4,0)$ theory, there are 272 -form tensor fields, whose ghosts for ghosts are in (000)(0100). We take a field $\Psi$ in (000)(0100) and also impose $\Psi \approx \Psi+\left.(\lambda \varrho)\right|_{(000)(0100)}$, for any $\varrho(x, \theta, \lambda)$ in (010)(1000). This type of "shift symmetry" is described in detail in ref. [25]. In terms of the terminology of ref. [26], one would say that $\Psi$ is not a function on the pure spinor space, but belongs to a section of a sheaf over pure spinor space.

Now, the calculation of the zero-mode cohomology is purely algebraic, and can be performed on a computer. The result is displayed in table 1. With a conformal assignment of dimensions, the scalars should have dimension 2, which means that $\Psi$ has dimension 0 . The superfields at different lower numbers are shifted downward, so that fields in the same row has the same dimension. Since $\lambda$ has dimension $-\frac{1}{2}$, the superfield at ghost number $-2+n$ has dimension $\frac{n}{2}$. Black dots in the table denotes the absence of cohomology.

Coordinate dependence of the fields in these modules will be related by derivatives in the full cohomology. Obviously, fields in different $C_{4} \approx \operatorname{USp}(8)$ R-symmetry modules do not talk to each other in the linear theory. From table 1, we can thus read the sequence of ghosts and fields for each of the fields. The action of the BRST operator (the nilpotent 


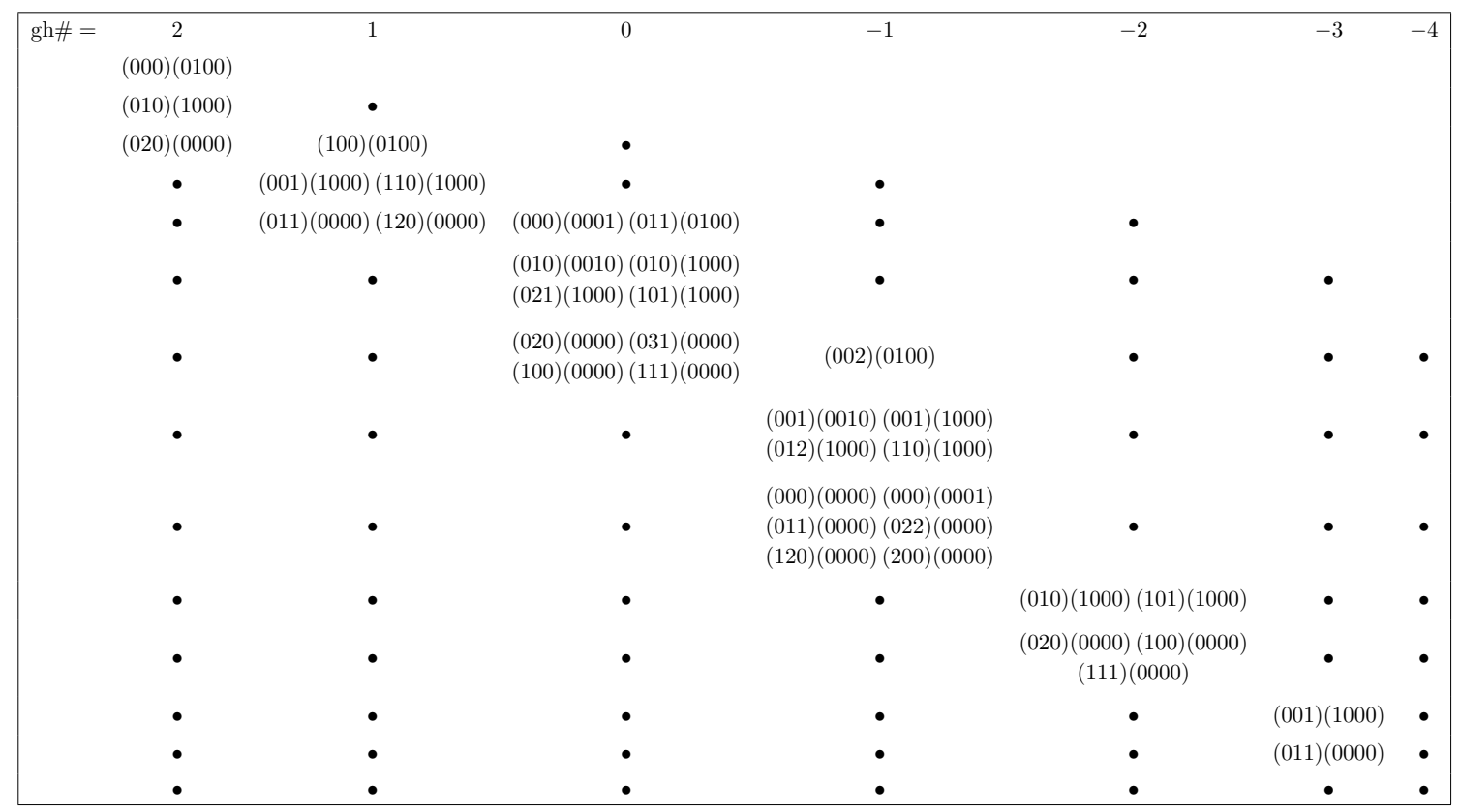

Table 1. The zero-mode cohomology in $\Psi$. The superfields at different ghost numbers are shifted so that fields in the same row has the same dimension. Black dots denote the absence of cohomology.

arrows in the following sequences) takes us one step to the right and two down. For the fields in $(0100)=\mathbf{2 7}$ we read off the sequence

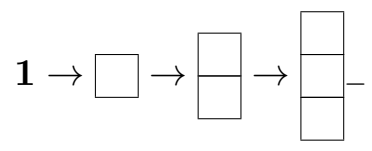

interpreted as the ghost for ghost, the ghost, the $B$-field, and its antifields or equation of motion $F_{-}=0$. Here, the convention is

$$
(020)=\square+, \quad(002)=\square-.
$$

The scalars in $(0001)=\mathbf{4 2}$ have their antifields in the same module at the correct dimension. The spinors in $(0010)=\mathbf{4 8}$ are found at the right dimension, with their equation of motion. The "gravitino" sequence in $(1000)=8$ is

$$
\alpha \rightarrow \square \otimes \alpha \rightarrow \square \otimes \alpha \rightarrow \square \otimes \alpha \ominus(030) \rightarrow(\square \otimes \alpha)^{\prime} \rightarrow(\square \otimes \alpha)^{\prime}
$$

where $\alpha=(010)$, and the "gravity" sequence in $(0000)=\mathbf{1}$ is

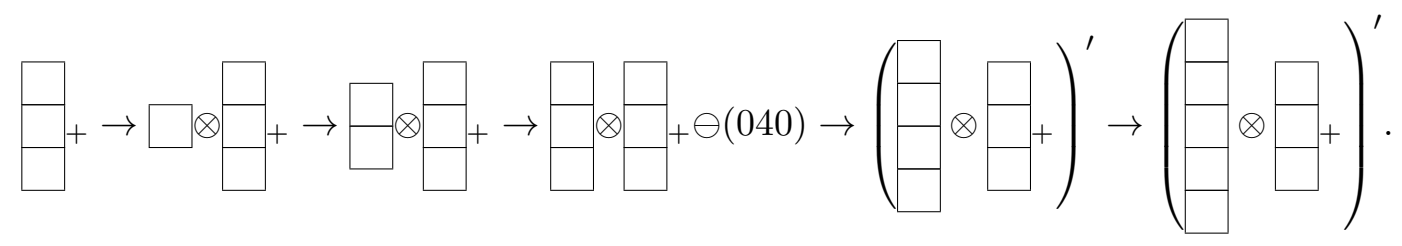


In both these cases, the equations of motion are the exterior derivative on the fields, except for the modules (030) and (040), respectively, which instead are surviving gauge invariant field strengths (this is indeed true also for the tensor fields, where the invariant field strength is in (020)(0100)). The primed tensor product in the "syzygies" (the reducibilities of the equations of motion, in two steps) implies a projection on the irreducible modules which are not reached via (030) or (040), respectively, and the arrow is nilpotent. So, by definition,

$$
\begin{gathered}
(\square \otimes \alpha)^{\prime}=\square \otimes \alpha \ominus(030) \\
(\square \otimes \square+)^{\prime}=\square \otimes \square+\ominus(040) .
\end{gathered}
$$

It is straightforward to verify that in the gravitino sequence,

$$
\begin{aligned}
& (\square \otimes \alpha)^{\prime}=(010) \oplus(101)=\square \otimes \bar{\alpha}, \\
& \left(\begin{array}{l}
\square \\
\square
\end{array}\right)^{\prime}=(001)=\bar{\alpha}, \\
& \left.(\square, \square)^{\prime}\right)^{\prime}=0,
\end{aligned}
$$

and in the gravity sequence,

$$
\begin{aligned}
& \left(\square \otimes \square+\square^{\prime}=(020) \oplus(100) \oplus(111),\right. \\
& \left(\square \otimes \square+\square^{\prime}=(011)\right. \text {, } \\
& (\square \otimes \square+)^{\prime}=0,
\end{aligned}
$$


To be concrete, the exotic graviton has ghosts for ghosts in $\exists_{+}$, ghosts in $\square \otimes \Theta_{+}$, gauge potentials in $\boxminus \otimes \theta_{+}$and equations of motion in $\boxminus \otimes \theta_{+} \ominus(040)$, where $\bigoplus_{+}=(040)$ is the module of the exotic Weyl tensor. The relation to the superfields of ref. [27] is not obvious. They start from representations of the superconformal group. The gauge invariant fields they list is of course in complete agreement (scalars in (000)(0001), spinors in (010)(0010), tensor field strength in (020)(0100), "gravitino" field strength in (030)(1000) and "gravity field strength" in (040)(0000)), but it is not clear how they in turn are built from potentials, since they appear in a gauge-invariant superfield starting with the scalars. In this context, the present superspace treatment might be interesting, in that it displays all the gauge symmetries and fields. The superfield at ghost number 0 has the scalars at $\theta^{2}$.

\section{$3 \quad N=(3,1)$ and $N=(3,0)$}

One might expect the construction of superfields for the $N=(3,1)$ model and the nonchiral $N=(2,2)$ supergravity should follow the same principles, where the field transforms as the ghosts of highest ghost number. The pure spinor superfield $\Psi$ for the $N=(3,1)$ model would transform in the module $(100)(1)$ of the R-symmetry $\operatorname{USp}(6) \times \operatorname{USp}(2)$, which is the module of the 12 chiral 2-form fields. However, I have not been able to perform such a construction with manifest $N=(3,1)$ supersymmetry, only $N=(3,0)$. Consider the $N=(3,1)$ supermultiplet. It contains the physical degrees of freedom, ${ }^{1}$ labelled by dimensions of modules of the little group $\mathrm{SU}(2) \times \mathrm{SU}(2) \times \mathrm{USp}(6) \times \mathrm{USp}(2)$,

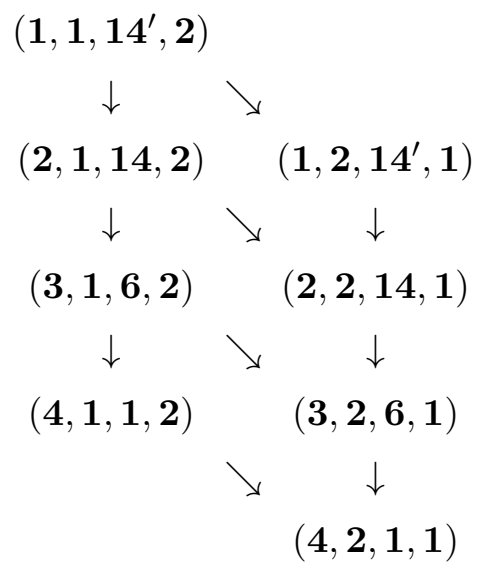

Here, $\mathbf{1 4}=(010)$, the $\epsilon$-traceless antisymmetric 2-index tensor, and $\mathbf{1 4}^{\prime}=(001)$, the $\epsilon$ traceless antisymmetric 3-index tensor. Vertical arrows indicate the action of the 3 chiral supersymmetries, increasing spin, and diagonal arrows of the single anti-chiral supersymmetry. The two columns form $N=(3,0)$ supermultiplets, which we may call the (exotic) $N=(3,0)$ gravitino and gravity multiplets. Note that the full $\operatorname{Spin}(1,5) \times \operatorname{USp}(6) \times \operatorname{USp}(2)$ is unbroken by this decomposition. The gravitino multiplet contains the scalars in $\left(\mathbf{1 4}^{\prime}, \mathbf{2}\right)$, which parametrise the coset $F_{4(4)} /(\mathrm{USp}(6) \times \mathrm{USp}(2))$, chiral spinors in $(\mathbf{1 4}, \mathbf{2})$, chiral 2 forms in $(\mathbf{6}, \mathbf{2})$ and an chiral exotic gravitino in $(\mathbf{1}, \mathbf{2})$. The gravity multiplet contains

\footnotetext{
${ }^{1}$ The module $(\mathbf{4}, \mathbf{1}, \mathbf{1}, \mathbf{2})$ is missing in the list of ref. [2].
} 


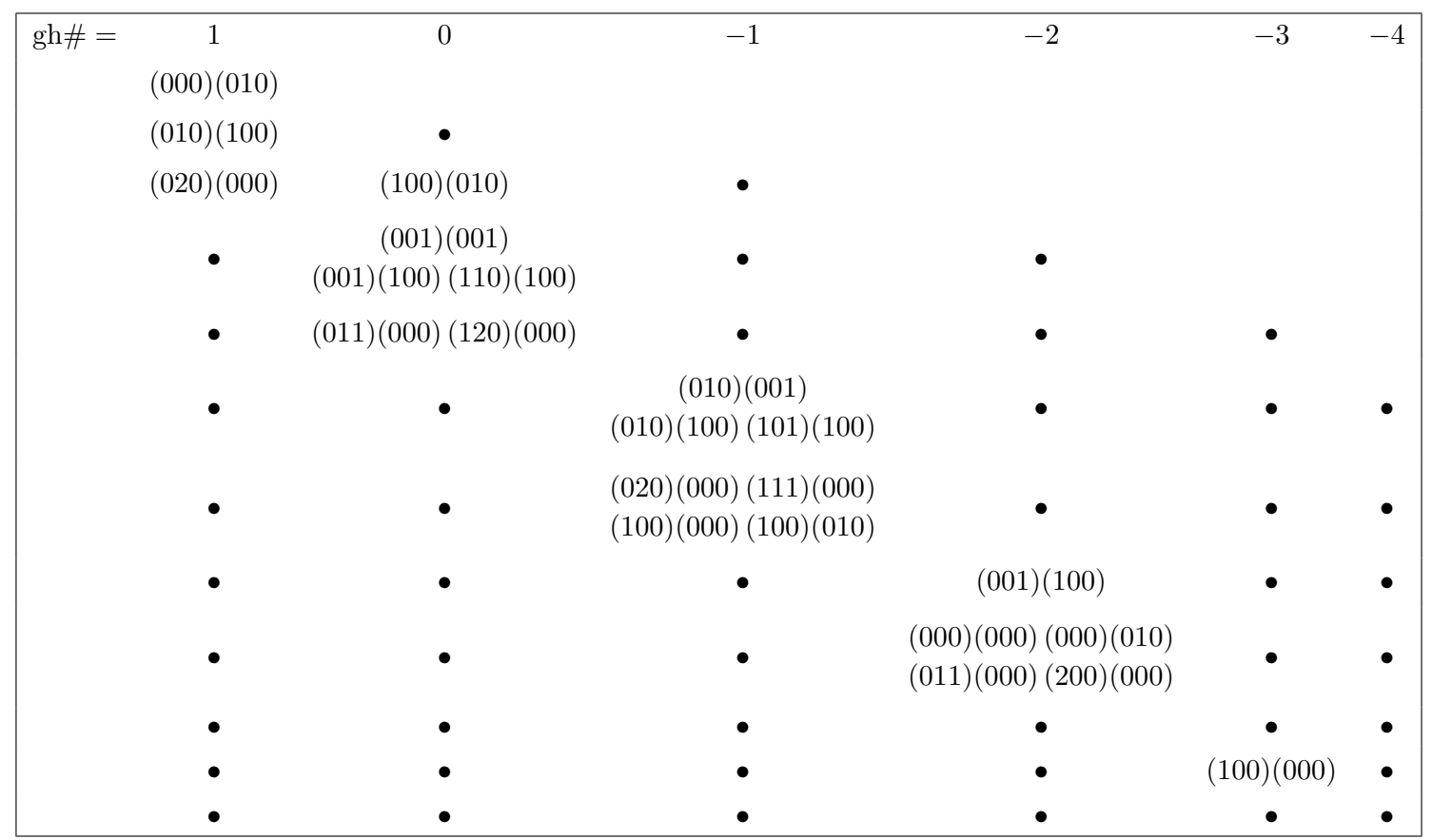

Table 2. The zero-mode cohomology for the $N=(3,0)$ gravity multiplet.

anti-chiral spinors in $\left(\mathbf{1 4}^{\prime}, \mathbf{1}\right)$, vector fields in $(\mathbf{1 4}, \mathbf{1})$, chiral gravitini in $\left(\mathbf{6}^{\prime}, \mathbf{1}\right)$ and a "semiexotic" gravity field. For these two $N=(3,0)$ multiplets it is straightforward to construct superfields along the same lines as in section 2. The "pure spinor" $\lambda$ transforms in $(010)(100)$ of $\operatorname{Spin}(1,5) \times \mathrm{USp}(6)$, and obeys $\left.\lambda^{2}\right|_{(100)(000)}=0$. The constraints are again irreducible. The gravitino multiplet (suppressing the $\operatorname{USp}(2)$ doublet, which is common to all fields in the multiplet) is given by a pure spinor superfield of ghost number 2 in $(000)(100)=(\mathbf{1}, \mathbf{6})$ with a shift symmetry in (010)(000). Its leading component is the second order ghost for the tensor field. The gravity multiplet is given by a field of ghost number 1 in (000)(010), with the ghosts for the gauge fields as leading part. The zero-mode cohomologies are given in tables 2 and 3.

From the tables, we read the sequences of ghosts fields and verify that the description is correct. The new information is the sequence for the "semi-exotic" $N=(3,1)$ graviton, which becomes

$$
\boxminus_{+} \rightarrow \square \otimes \boxminus_{+} \rightarrow \boxminus \otimes \boxminus_{+} \ominus(031) \rightarrow\left(\boxminus \otimes \boxminus_{+}\right)^{\prime} \rightarrow\left(\boxminus \otimes \boxminus_{+}\right)^{\prime}
$$

where ()$^{\prime}$ now indicates the removal of modules coming through $\bigoplus_{+}=(031)$, the module of the "semi-exotic Weyl tensor".

Going back to $N=(3,1)$, one might have suspected that the cohomologies of the $N=(3,0)$ gravity and gravitino multiplets would combine into the cohomology of an $N=(3,1)$ superfield transforming in $(000)(100)(1)$. One should then use a "pure spinor" $\lambda$ in $(010)(100)(0) \oplus(001)(000)(1)$. Surprisingly, it turns out that such a field seems to become "topological", in the sense that all sequences of zero-mode cohomologies (ghosts 


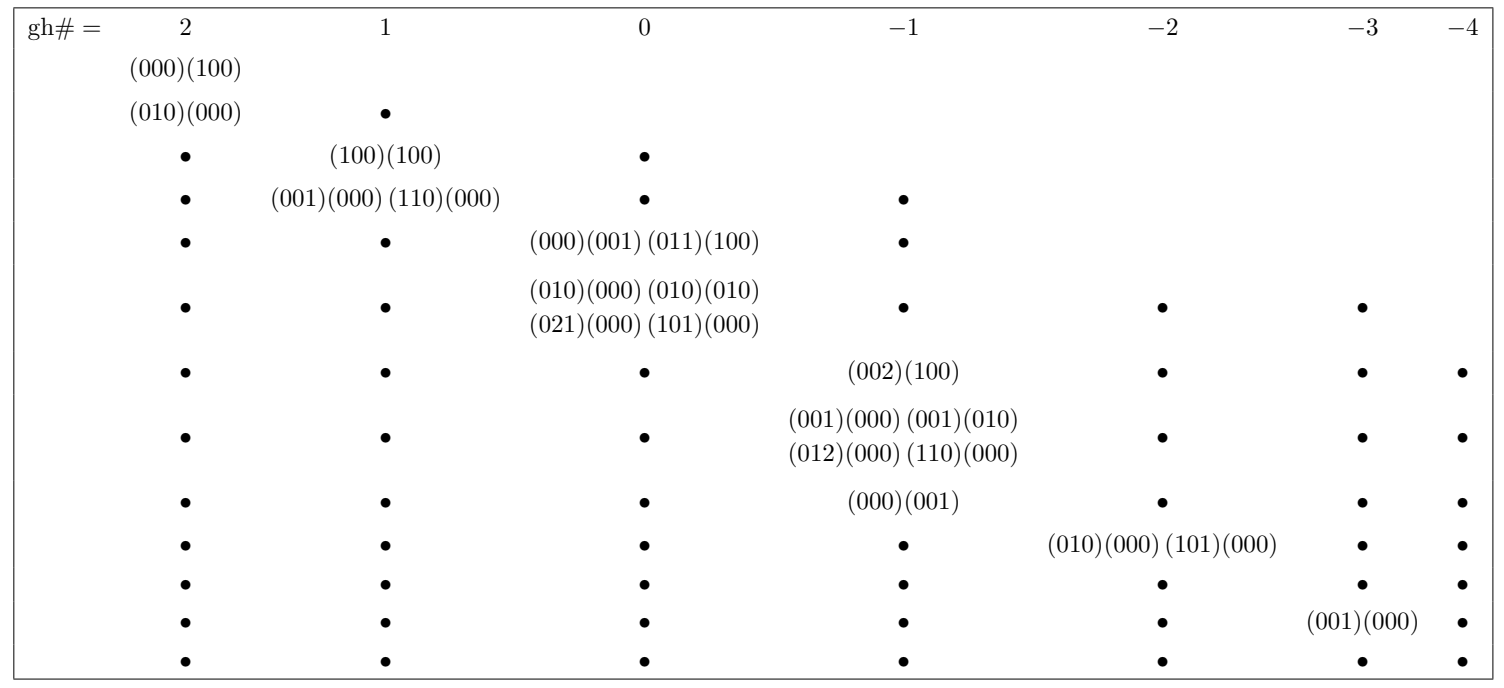

Table 3. The zero-mode cohomology for the $N=(3,0)$ gravitino multiplet.

and component fields) consist of the tensor product of some modules (the ghost number 2 ghosts of the $N=(3,0)$ gravitino multiplet) with forms of increasing degree, and no local degrees of freedom remain. I have no clear explanation of this. An investigation of $N=(2,2)$ supergravity might provide some insight.

\section{Conclusions}

This note has presented superfields for the $N=(4,0)$ and $(3,1)$ exotic supergravity multiplets in $D=6$, in the latter case in terms of $N=(3,0)$ superfields.

It is not be possible to write a covariant action reproducing $Q \Psi=0$ (only). Noncovariant component actions are given in refs. [4, 5]. A pseudo-action, where some fields need to be (consistently) set to 0 , should exist. Then one would first need to extend the pure spinor superspace to include non-minimal variables [28]. This enables non-degenerate integration, and the procedure is standard [22]; the integration measure corresponds to the top cohomology of the singlet superfield of eq. (2.2). One would also need a "conjugate" pure spinor superfield $\Psi^{\star}$, which (for the $N=(4,0)$ model) would presumably transform in the module (011)(0000), since this is the highest cohomology in $\Psi$. The cohomology in $\Psi^{\star}$ is conjugate to the one in $\Psi$. And, it is necessary to show that integration, with some $\lambda$ 's inserted, implies the proper shift symmetries of $\Psi$ and $\Psi^{\star}$. All this remains yet to be done, but it is doubtful that it would contribute significantly to the understanding of the model. The corresponding procedure for the $N=(2,0)$ tensor multiplet works [29], and yields two tensor multiplets of the same chirality, of which one can be set to zero.

Also without a pseudo-action, one may discuss possible deformations of $Q \Psi=0$. Such considerations are usually especially powerful in a pure spinor superfield framework, since all gauge symmetries and equations are treated simultaneously, and the field carries as low dimension and as high ghost number as possible. However, naïve dimensional 
considerations point at the impossibility of finding local interactions this way, just as they do for the $N=(2,0)$ theory (without the introduction of some dimensionful constant).

\section{Acknowledgments}

The author would like to thank Marc Henneaux, Victor Lekeu, Jakob Palmkvist and Ingmar Saberi for discussions on various aspects of the models and the formalism.

Open Access. This article is distributed under the terms of the Creative Commons Attribution License (CC-BY 4.0), which permits any use, distribution and reproduction in any medium, provided the original author(s) and source are credited.

\section{References}

[1] J.A. Strathdee, Extended Poincaré Supersymmetry, Int. J. Mod. Phys. A 2 (1987) 273 [INSPIRE].

[2] C.M. Hull, Strongly coupled gravity and duality, Nucl. Phys. B 583 (2000) 237 [hep-th/0004195] [INSPIRE].

[3] C.M. Hull, Symmetries and compactifications of $(4,0)$ conformal gravity, JHEP 12 (2000) 007 [hep-th/0011215] [INSPIRE].

[4] M. Henneaux, V. Lekeu and A. Leonard, The action of the (free) (4,0)-theory, JHEP 01 (2018) 114 [Erratum JHEP 05 (2018) 105] [arXiv:1711.07448] [INSPIRE].

[5] M. Henneaux, V. Lekeu, J. Matulich and S. Prohazka, The Action of the (Free) $\mathcal{N}=(3,1)$ Theory in Six Spacetime Dimensions, JHEP 06 (2018) 057 [arXiv:1804.10125] [InSPIRE].

[6] R. Minasian, C. Strickland-Constable and Y. Zhang, On symmetries and dynamics of exotic supermultiplets, JHEP 01 (2021) 174 [arXiv:2007.08888] [INSPIRE].

[7] M. Günaydin, Unified non-metric $(1,0)$ tensor-Einstein supergravity theories and $(4,0)$ supergravity in six dimensions, arXiv:2009.01374 [INSPIRE].

[8] Y. Bertrand, S. Hohenegger, O. Hohm and H. Samtleben, Toward exotic 6D supergravities, Phys. Rev. D 103 (2021) 046002 [arXiv: 2007.11644] [InSPIRE].

[9] O. Hohm and H. Samtleben, Exceptional Field Theory I: $E_{6(6)}$ covariant Form of M-theory and Type IIB, Phys. Rev. D 89 (2014) 066016 [arXiv:1312.0614] [InSPIRE].

[10] M. Cederwall and J. Palmkvist, Extended geometries, JHEP 02 (2018) 071 [arXiv: 1711.07694] [INSPIRE].

[11] N. Berkovits, Super Poincaré covariant quantization of the superstring, JHEP 04 (2000) 018 [hep-th/0001035] [INSPIRE].

[12] N. Berkovits, Cohomology in the pure spinor formalism for the superstring, JHEP 09 (2000) 046 [hep-th/0006003] [INSPIRE].

[13] M. Cederwall, B.E.W. Nilsson and D. Tsimpis, Spinorial cohomology and maximally supersymmetric theories, JHEP 02 (2002) 009 [hep-th/0110069] [INSPIRE].

[14] M. Cederwall, $N=8$ superfield formulation of the Bagger-Lambert-Gustavsson model, JHEP 09 (2008) 116 [arXiv:0808.3242] [INSPIRE]. 
[15] M. Cederwall, Superfield actions for $N=8$ and $N=6$ conformal theories in three dimensions, JHEP 10 (2008) 070 [arXiv: 0809. 0318] [INSPIRE].

[16] M. Cederwall, Towards a manifestly supersymmetric action for 11-dimensional supergravity, JHEP 01 (2010) 117 [arXiv:0912.1814] [INSPIRE].

[17] M. Cederwall, D=11 supergravity with manifest supersymmetry, Mod. Phys. Lett. A 25 (2010) 3201 [arXiv: 1001.0112] [INSPIRE].

[18] M. Cederwall, Pure spinor superspace action for $D=6, N=1$ super-Yang-Mills theory, JHEP 05 (2018) 115 [arXiv:1712.02284] [INSPIRE].

[19] M. Cederwall, An off-shell superspace reformulation of $D=4, N=4$ super-Yang-Mills theory, Fortsch. Phys. 66 (2018) 1700082 [arXiv:1707.00554] [INSPIRE].

[20] M. Movshev and A.S. Schwarz, On maximally supersymmetric Yang-Mills theories, Nucl. Phys. B 681 (2004) 324 [hep-th/0311132] [INSPIRE].

[21] M. Movshev and A.S. Schwarz, Supersymmetric Deformations of Maximally Supersymmetric Gauge Theories, JHEP 09 (2012) 136 [arXiv:0910.0620] [INSPIRE].

[22] M. Cederwall, Pure spinor superfields - an overview, in Breaking of Supersymmetry and Ultraviolet Divergences in Extended Supergravity, Springer Proceedings in Physics 153, Springer, Cham Switzerland (2014), pp. 61-93 [arXiv:1307.1762] [INSPIRE].

[23] M. Cederwall and J. Palmkvist, Superalgebras, constraints and partition functions, JHEP 08 (2015) 036 [arXiv : 1503.06215] [inSPIRE].

[24] M. Cederwall, J. Palmkvist and I. Saberi, work in progress.

[25] M. Cederwall and A. Karlsson, Pure spinor superfields and Born-Infeld theory, JHEP 11 (2011) 134 [arXiv:1109.0809] [INSPIRE].

[26] R. Eager, I. Saberi and J. Walcher, Nilpotence varieties, arXiv:1807.03766 [INSPIRE].

[27] M. Chiodaroli, M. Günaydin and R. Roiban, Superconformal symmetry and maximal supergravity in various dimensions, JHEP 03 (2012) 093 [arXiv: 1108.3085] [INSPIRE].

[28] N. Berkovits, Pure spinor formalism as an $N=2$ topological string, JHEP 10 (2005) 089 [hep-th/0509120] [INSPIRE].

[29] M. Cederwall, unpublished. 